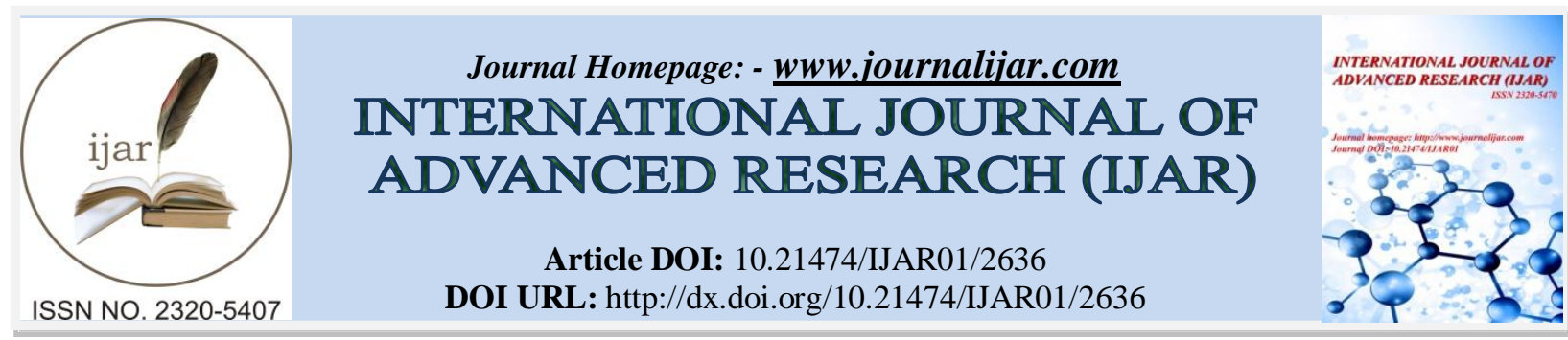

RESEARCH ARTICLE

\title{
VATS ASSISTED REPOSITIONING OF A CENTRAL VENOUS ACCESS DEVICE CATHETER CAUSING INADVERTENT SVC PUNCTURE: A RARE CASE REPORT AND LITERATURE REVIEW.
}

\author{
Dr. Dhananjay B Ghuge, Dr. Subraharsh Singh, Dr. Alok Tiwari, Dr. Kalpana Munde, Dr. Aviral Verma and \\ Dr. B.N. Naik
}

\section{Manuscript Info}

\section{Manuscript History}

Received: 30 October 2016

Final Accepted: 29 November 2016

Published: December 2016

\begin{abstract}
Central Venous Access Device (CVAD) is commonly used in cancer patients for administration of chemotherapy. Malpositioning of venous access port catheter in to the contra lateral pleural cavity is a rare possibility, but can have potentially catastrophic complications. The treatment of choice is immediate removal or repositioning of the catheter by either surgery or endovascular interventions. We herein report an interesting case of a 53 year old female, a case of Ca Right breast that had malpositioning of venous access port catheter through SVC (Superior Vena Cava) into contra lateral pleural cavity. In this article we have discussed the use of Video Assisted Thoracic Surgery (VATS) as well as review of the existing literature.
\end{abstract}

Copy Right, IJAR, 2016,. All rights reserved.

\section{Introduction:-}

Long term Central Venous Access Devices (CVAD) are frequently utilized in oncological practice, most commonly for chemotherapy and long term parenteral nutrition. CVAD insertion can be done using the Scheldinger's technique or under radiological guidance, either by ultrasound or fluoroscopy. ${ }^{1}$

Malpositioning of the CVAD is a known complication with reported incidence ranging from less than $1 \%$ to more than $60 \%{ }^{2 .}$ Unfortunately, improper catheter tip position is associated with a high rate of complications. ${ }^{3}$

Various methods of repositioning are described in the literature, which includes direct manipulation by guide wire or tip-deflecting wire manipulation via transfemoral venous approach and by injection of contrast or saline. ${ }^{4}$

We hereby report an interesting case; presented as sudden catastrophe, demonstrating malpositioning of catheter tip. She underwent venous access port insertion without image guidance along with a planned elective procedure of Right MRM (Modified Radical Mastectomy) under general anaesthesia. Patient landed up with massive haemothorax due to dilator with sheath piercing superior vena cava (SVC) into the contra lateral pleural cavity. Tip of the catheter inserted through this sheath was then protruding into the right pleural cavity.

Case Report: A 53 yrs old female patient presented to our hospital with a lump in right breast since 2 month duration. After all routine and metastatic work up, she was staged as clinically operable locally advanced breast cancer T3N1M0 (stage IIIA). Informed consent regarding Right MRM and Left sided venous access port insertion in same sitting for further chemotherapy was taken. We routinely practice venous access port insertion on the contralateral side followed by definitive surgery, Right MRM in this case.

We usually insert CVAD without image guidance, guided by the cardiac ectopics on the ECG monitor, elicited when guide wire reaches the heart to assess the proper position of the guide wire. It is observed to be accurate in nearly $98 \%$ of the cases. We remove the guide wire keeping venous cannula in-situ and re-insert it if proper ectopics are not elicited on the ECG monitor. 
In this case, during left sided venous access port insertion with standard Scheldinger's technique without image guidance, proper cardiac ectopics were noted in the ECG. However after introducing the dilator sheath patient suddenly developed hypotension with fall in $\mathrm{O}_{2}$ saturation. Left sided pneumothorax was suspected as the procedure was being done on the left side. A left sided ICD (Inter Costal Drainage) tube was inserted on table, but there was no evidence of pneumothorax or haemothorax. In view of persistent hypotension and falling $\mathrm{O}_{2}$ saturation Right MRM procedure was deferred and patient was shifted to the ICU for stabilization. Chest X-ray was done which showed massive Right sided haemothorax and abnormal positioning of catheter tip (Fig 1).

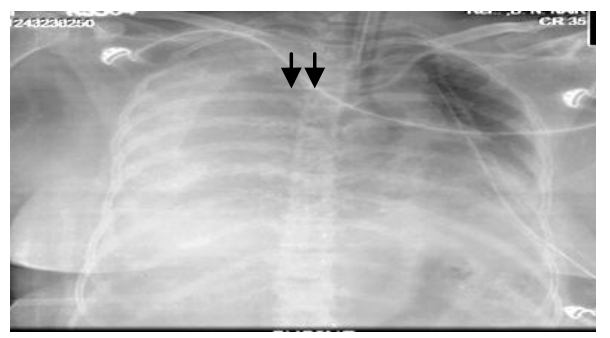

Fig.1:- Showing Right side hemothorax with abnormal catheter tip (black arrows) of left sided venous access port.

Immediate Right side ICD insertion done and haemothorax of approximately $1300 \mathrm{ml}$ drained. After stabilizing the patient a contrast CT Thorax was done which revealed position of catheter tip in the right pleural cavity piercing through SVC (3 cm) (Fig 2). Case was discussed with interventional cardiologists. They suggested varied opinions like stenting of SVC, Simple removal of CVAD, angiographic repositioning or angiographic balloon assisted repositioning of the catheter. As patient had to undergo MRM, a combined procedure under vision with VATS was deemed more appropriate to resolve this situation. Patient was taken up for surgery with the plan of repositioning of catheter with suturing of SVC rent if needed and Right MRM under GA. Patient was then subjected to VATS with single lung ventilation and Right side thoracoscopy was done. Catheter tip was seen in Right pleural cavity (Fig 3).

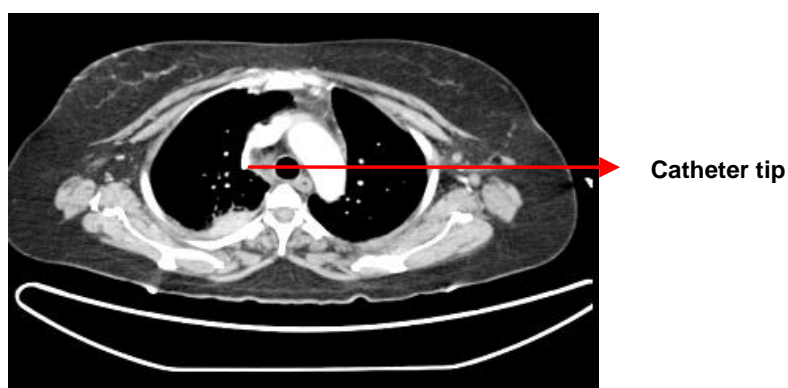

Fig. 2:- CT Thorax showing catheter tip piercing SVC and located in Right pleural cavity.

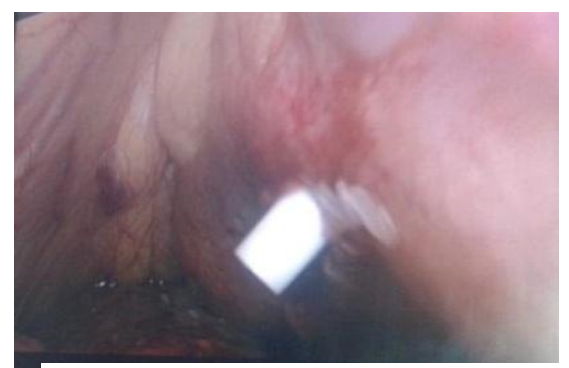

Fig. 3:- Thoracoscopic view showing catheter tip in right pleural cavity.

Left sided venous access port was released from the subcutaneous pouch made previously on the left infraclavicular region. The catheter was pulled back for 3 to $4 \mathrm{~cm}$. Meanwhile under thoracoscopic vision pressure was applied with a gauze piece through working port at catheter exit site on SVC. Pressure was kept for 5 minutes and then observed for any active bleeding. There was no active bleeding on observation for 15-20 minutes (Fig 4). CVAD was refashioned after reducing the catheter length by $3 \mathrm{~cm}$. and previously inserted right sided ICD was kept in situ. Check X- ray done showed catheter tip in left innominate vein with no evidence of haemothorax (Fig 5). Patient was discharged on $3^{\text {rd }}$ post operative day in a stable condition after ICD tube removal. Now she is recieving adjuvant treatment using the same venous access port for chemotherapy drug infusion without any problem. 


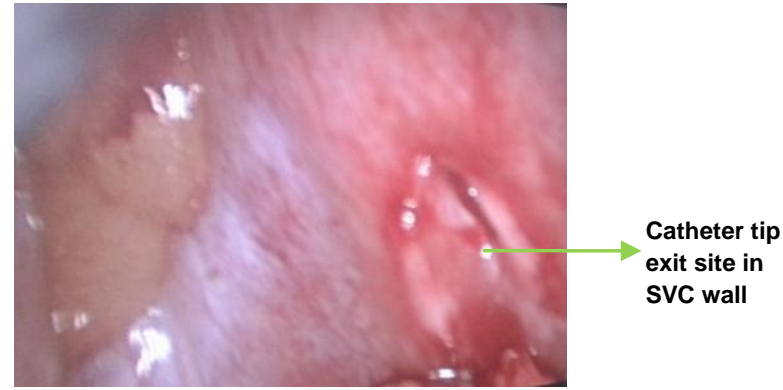

Fig 4:- Thoracoscopic view showing catheter tip exit site after repositioning of venous access port

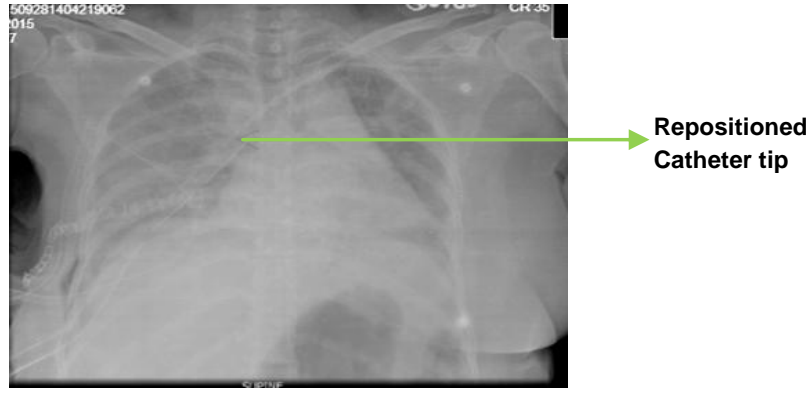

Fig 5:- Showing catheter in left innominate vein with right ICD in situ

\section{Discussion:-}

Insertion of Ports, Groshong or Hickman catheters is a fairly routine practice in Oncology to provide a permanent central venous access for infusional chemotherapy, parenteral nutrition or hemopoietic stem cell transplantation. Though radiological guidance in placing the CVAD has been demonstrated to result in smaller incidence of catheter malpositions, majority of the procedures are done by Scheldinger technique using anatomical landmarks. ${ }^{1}$

An implantable port device provides an easily accessible central route for long-term chemotherapy patients. Many immediate and late complications of implantable port catheters have been reported on. The early complications include pneumothorax, hematoma, malposition, embolism or arrhythmia, and these are often related to the placement technique. The delayed complications include skin necrosis, infection, catheter fracture, occlusion or thrombosis. ${ }^{5}$

Why do left-implanted catheters with high-lying tips represent such a risk? The first hypothesis concerns anatomy: venographies show that in most patients and especially in the stocky ones (i.e., those with a short, wide thorax), the left innominate vein forms a more marked angle with the vena cava. Therefore, when the surgeon pushes the catheter, it often strikes against the external wall of the vena cava at the level of this angle. Resistance may occur when the catheter is pushed downward, and the catheter guide wire may injure the endothelium. A case of a Hickman catheter perforating the wall of the superior vena cava into the pericardium has been reported by CathcartRake and Mowery. ${ }^{6}$

Malposition is one of the most common complication after catheter placement. Catheter tip may lie in Internal jugular vein, contralateral jugular, axillary or azygous vein. If the catheter is too long, the tip may coil in right atrium or inferior vena cava. Usually, fluoroscopic guidance prevents this complication. Even if catheter is placed at bedside catheter tip must be checked by a chest $\mathrm{x}$-ray. ${ }^{7}$

Malposition increases the risk of thrombosis and perforation. Therefore, an incorrect tip position must be amended as soon as possible. Besides simply exchanging the malpositioned catheter, there are interventional methods which may facilitate correct catheter positioning. ${ }^{8}$

Hence the displaced catheter either has to be removed and then re-inserted, or repositioned. Removal and reinsertion of the CVAD would entail another major procedure and its inherent risk in potentially immuno-compromised patients, hence repositioning of the same CVAD would appear to be a more attractive option.

Here, we reported an interesting case of malposition of venous access port catheter tip into the contralateral pleural cavity. To our knowledge, there is no similar case like ours reported till date in current medical literature in which VATS is used. The use of VATS helps in under vision repositioning of the catheter rather than simple pulling and allows for any haemostatic intervention like haemoclip application, suturing or simple pressure gauze application in case of bleeding through catheter exit site in the pleural cavity.

Perforation of central veins or right-sided cardiac chambers can be catastrophic. In clinical practice, it is often difficult to ascertain what caused the venous perforation; the introducer needle, the guidewire, or the dilator. The 
literature reports cases of guidewire-related perforation of the great vessels including the brachiocephalic and subclavian veins. This important complication occurs when excessive force is applied against resistance when introducing the guidewire, especially if the straight or angle tip wire, rather than $\mathrm{J}$ tip style wire, is used. In most instances, bleeding from a small penetrating hole in a vein will stop spontaneously by vasospasm or by external compression of the surrounding tissues. However, serious cases of hemothorax, including mortality, due to the above complication have been reported. Making a timely diagnosis in such cases requires maintaining a high index of suspicion when there is an unexplained drop in hemoglobin or the development of unilateral pleural effusion ipsilateral to a recently placed or attempted central venous catheterization. Treatment of a serious perforation may necessitate the insertion of a chest tube or an emergent thoracotomy ${ }^{9}$.

Left-sided catheters have been reported to have a higher incidence of vascular perforation. In a retrospective review of more than 1,000 catheter placements, Mukau et $\mathrm{al}^{10}$ reported four patients with perforation of the SVC. All four had left-sided catheters and the average time interval to clinical symptoms was 5 days.

Tocino and Watanabe ${ }^{11}$ described nine catheter-related perforations of the SVC. Eight of these nine patients had left sided catheters. Similarly, Duntley and colleagues ${ }^{12}$ reported a series of eight patients who had catheter-related vascular perforations. Seven of these catheters were inserted from the left side, and six of these patients had catheter tips that were described as "abutting the right lateral wall of the superior vena cava." In this group of eight patients, the clinical manifestations of vascular perforation became evident 2.9 days 0.8 after catheter insertion.

\section{Conclusion:-}

The most feared catheter-related complications, such as vascular perforation and cardiac arrhythmias, are rare. Importantly, these complications are often caused by physician errors during catheter insertion procedures. In such circumstances ipsilateral as well as contralateral injury should be kept in mind. The incidence of vascular injuries are more with left-sided catheters or if the catheter tip is abutting a vascular wall. Therefore, the preferred insertion should be on the right side whenever possible and fluoroscopy guidance should be used to visualize all endovascular manipulations during the catheter insertion procedure. For the malpositioned catheter in thoracic cavity, VATS guided repositioning may be considered as good alternative option.

\section{References:-}

1. Marcy PY. Central venous access: Techniques and indication in oncology. Eur Radiol 2008; 18: 2333-44.

2. Chauhan A. Malpositioning of central venous catheter: Two case reports. Indian J Anaesth 2008; 52: 337-9.

3. De Chicco R, Seidner DL, Brun C, Steiger E, Stafford J, Lopez R. Tip position of long-term central venous access devices used for parenteral nutrition. JPEN J Parenter Enteral Nutr 2007; 31: 382-7.

4. Hartnell GG, Roizental M. Percutaneous transfemoral repositioning of malpositioned central venous catheters. AJR Am J Roentgenol 1995; 164: 1003-6.

5. Ballarini C, Intra M, Pisani Ceretti A, Cordovana A, Pagani M, Farina G et al. Complications of subcutaneous infusion port in the general oncology population. Oncology. 1999; 56: 97-102.

6. Cathcart-Rake WF, Mowery WE. Intrapericardial infusion of 5-fluorouracil.An unusual complication of a Hickman catheter. Cancer 1991; 67735-7.

7. Funaki B. Central venous access: a primer for the diagnostic radiology.AJR Roentgenol, 2002; 179:309-18.

8. Teichgraber UK, et al. Central venous access catheters: radiological management of complications. Cardiovasc Intervent Radiol.2003;26:321-33

9. Y. Innami, T. Oyaizu, T. Ouchi, N. Umemura, and T. Koitabashi, "Life-threatening hemothorax resulting from right brachiocephalic vein perforation during right internal jugular vein catheterization, "Journal of Anesthesia, 2009; 23(1):135-38.

10. Mukau L, Talamini MA, Sitzmann JV. Risk factors for central venous catheter-related vascular erosions. J Parenter Enteral Nutr 1991; 15:513-16.

11. Tocino IM, Watanabe A. Impending catheter perforation of superior vena cava: radiographic recognition. AJR Am J Roentgenol 1986; 146:487-90.

12. Duntley P, Siever J, Korwes ML, Harpel K, Heffner JE. Vascular erosions by central venous catheters. Clinical features and outcome. Chest 1992; 101: 1633-38. 